\title{
Self-Organization of Wireless Networks: The New Frontier (Keynote Speech)
}

\author{
Sudhir Dixit \\ Nokia Research, Burlington, MA, USA \\ sudhir.dixit@nokia.com
}

\begin{abstract}
The size of the internet will increase with the mainstream adoption of the broadband mobility connecting a myriad of devices and sensors at homes and businesses and the use of IPv6. All this will add to the spatio-temporal complexity of the network topology and dynamics. We present a brief overview of the role that self-organization can play in this new era of complexity. Issues of QoS, scalability, robustness, and reachability, among others (e.g., heterogeneity) will dominate the research in the future. First, we present the definition, scope, and applicability of self-organization. Then we briefly articulate the need for selforganization, and some recent breakthrough advances in this emerging area of research. This is followed by some near- and long-term scenarios where self-organization can be applied, and some results that we have obtained. We conclude the talk with a discussion on the key challenges that lie ahead.
\end{abstract}

